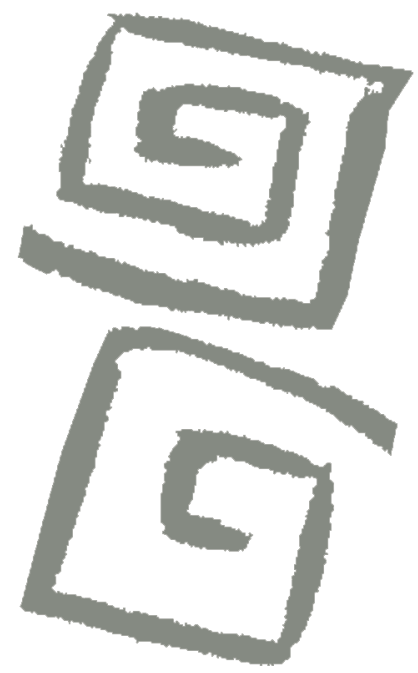

\title{
El uso de "virado" como estrategia de reducción de daños entre los usuarios de crack del estado de Pernambuco, Brasil
}

\author{
The use of "virado" as a harm reduction strategy among \\ crack users in the State of Pernambuco, Brazil
}

Renata Barreto Fernandes de Almeida', Naíde Teodósio Valois Santos², Ana Maria de Brito ${ }^{3}$, Keila Silene de Brito e Silva ${ }^{4}$, Iracema de Jesus Almeida Alves Jacques ${ }^{5}$, Solange Aparecida Nappo ${ }^{6}$

${ }^{1}$ Autora de correspondencia. Psicóloga. Doctora en Medicina Preventiva. Profesora titular, Centro Universitário do Vale do Ipojuca, Recife, Pernambuco, Brasil. $\triangle$ iD

${ }^{2}$ Médica sanitarista. Doctora en Salud Pública. Investigadora, Instituto Aggeu Magalhães, Fundação Oswaldo Cruz, Recife, Pernambuco, Brasil. $\square$ iiD

${ }^{3}$ Médica. Doctora en Salud Pública. Investigadora, Instituto Aggeu Magalhães, Fundação Oswaldo Cruz, Recife, Pernambuco, Brasil. $\bowtie$ (iD)

${ }^{4}$ Fonoaudióloga. Doctora en Salud Pública. Profesora, Centro Acadêmico de Vitória, Universidade Federal de Pernambuco, Pernambuco, Brasil. $₫$ (iD)

${ }^{5}$ Enfermera. Doctoranda en Salud Pública, Instituto Aggeu Magalhães, Fundação Oswaldo Cruz, Recife, Pernambuco, Brasil. $\square$ (iD)

${ }^{6}$ Farmacéutica. Doctora en Psicobiología. Profesora Adjunta, Universidade Federal de São Paulo, São Paulo, Brasil. $\square$ (iD)
RESUMEN El objetivo del estudio es discutir uno de los usos del crack denominado "virado", como estrategia de reducción de daños entre las personas que usan crack en Pernambuco, Brasil. Se realizó una investigación cualitativa y transversal. Desde marzo hasta agosto de 2016, se realizaron entrevistas semiestructuradas sobre la cultura del uso de crack a 39 personas que usan esta substancia. El límite de participantes se estableció por el criterio de saturación. Los datos se analizaron con la técnica de análisis de contenido. Las personas que participaron relataron que el virado es una manera distinta de utilizar el crack y, al comparar su efecto con el uso fumado o inhalado, mencionaron que el virado produce menos impacto en las relaciones interpersonales y en la libido, además de reducir el uso compulsivo de crack, cuestiones que se podrían considerar como estrategias de reducción de daños. Un aspecto negativo es que comparten los canutos para aspirar el virado, lo cual es una situación de riesgo para la transmisión de enfermedades infecciosas. Conocer la cultura del uso del crack en distintas formas y situaciones es imprescindible para la planificación y desarrollo de acciones de atención a la salud. PALABRAS CLAVES Cocaína Crack; Usuarios de Drogas; Reducción del Daño; Brasil.

\begin{abstract}
The aim of the study is to discuss the use of crack in the form of "virado" as a harm reduction strategy in Pernambuco, Brazil. This is a cross-sectional study with a qualitative approach in which semi-structured interviews were conducted regarding aspects related to the culture of crack use with 39 crack users between March and August 2016. Participants were recruited using saturation criteria and data were analyzed through content analysis. Respondents discussed the use of "virado" and compared its effects in relation to crack, addressing improvement in interpersonal relationships, libido, and noncompulsive drug use, which can all be understood as harm reduction strategies. On the other hand, equipment sharing for the use of "virado" was identified as a high-risk practice with regards to the transmission of infectious diseases. Knowing about the culture of crack use in different contexts is essential in order to plan and develop health care actions.
\end{abstract}

KEY WORDS Crack Cocaine; Drug Users; Harm Reduction; Brazil. 


\section{INTRODUCCIÓN}

El consumo de crack surgió en Brasil a fines de la década de 1980, como un fenómeno de rápida expansión ${ }^{(1)}$, principalmente, entre la población con mayor vulnerabilidad social. Bastos y Bertoni ${ }^{(2)}$ refuerzan ese perfil y señalan que los usuarios de crack y/o de sustancias similares son, en su mayoría, hombres, negros, jóvenes, poco escolarizados, que están viviendo en situación de calle y que no tienen empleo o ingresos fijos.

En el campo de la salud, podemos encontrar, además, problemas asociados al consumo de drogas -en especial, del crack- como, por ejemplo, mayor exposición a la infección por $\mathrm{VIH}$ y otras enfermedades sexualmente transmisibles, accidentes de tránsito, sobredosis, enfermedades cardiorrespiratorias, y agravios relacionados con la violencia ${ }^{(3,4)}$. Así, el potencial de daños causados por el crack es bastante elevado, sumados a los daños causados por la propia sustancia, por lo que su abuso se transformó en un problema en Brasil(5).

De acuerdo con Bastos y Bertoni ${ }^{(2)}$, al comparar los usuarios de crack con los de cocaína intranasal, los primeros parecen tener un patrón más grave de consumo, mayor vinculación con actividades ilegales, mayores riesgos de efectos adversos al uso de cocaína, mayores vínculos con la prostitución y más chances de vivir o haber vivido en la calle.

En ese escenario, ante la necesidad de un tratamiento efectivo para las personas que enfrentaban problemas derivados del uso abusivo o de la dependencia de alguna sustancia psicoactiva, el uso problemático de drogas, en especial del crack, pasó a formar parte de la agenda de las políticas públicas de Brasil. Según Petuco ${ }^{(6)}$, durante muchos años, enfrentar las problemáticas derivadas del consumo de drogas "oscilaba entre una cuestión médica y un problema de carácter estrictamente social; por otro lado, había mucha rigurosidad con las drogas concebidas como ilícitas, y condescendencia con el alcohol".

Según el autor, en la actualidad, los profesionales que trabajan en el área reconocen la complejidad de los riesgos asociados al uso de drogas. Por mayores que sean las diferencias conceptuales entre diferentes investigadores y teóricos del tema, hay un consenso con relación a la necesidad de una atención integral, lo que siempre debe incluir sus familias y redes sociales. Otra cuestión importante señalada por el autor es que, al tratarse de un problema de difícil resolución, no deberían implementarse intervenciones unilaterales, sino que se requieren acciones conjuntas, que incluyan estrategias que involucren las áreas de salud, seguridad, deportes, cultura, educación, asistencia social, entre otras.

Hasta fines de la década de 1980, la orientación de las estrategias prohibicionistas vinculadas a la idea de "guerra contra las drogas" clausuraban el espacio para la reflexión o incluso la comprensión del consumo de drogas como un fenómeno multideterminado y con necesidad de intervenciones interdisciplinarias ${ }^{(7)}$.

Con la aparición del sida y la comprensión del concepto de vulnerabilidad, el proceso de enfermar ya no era solo el resultado de un conjunto de aspectos individuales, sino también colectivos y contextuales ${ }^{(8)}$. Así, las estrategias de reducción de daños tomaron cuerpo, y la prevención que se realizaba de forma exclusiva para el sida -por medio de los programas de intercambios de jeringasse amplió a la concepción de reducción de daños sociales y de la salud. Para Machado y Boarini $^{(7)}$, en ese momento se comenzó a concebir que las acciones de reducción de daños deberían ser planificadas de acuerdo con el contexto sociocultural en el que serían aplicadas.

Es importante también destacar que las estrategias de reducción de daños, en contraposición con las estrategias prohibicionistas de alta exigencia, se caracterizan por medidas de baja exigencia, dado que no definen como meta inicial -para el cuidado o incluso para el tratamiento- la abstinencia del uso de drogas. Además, establecen que la atención de la salud debe ir, preferencialmente, hasta el lugar donde se encuentra la persona, y no al contrario ${ }^{(9)}$.

La reducción de daños permite que la persona que hace un uso abusivo de las 
drogas participe de su proceso de cuidado, lo que la caracteriza como una medida formulada de abajo hacia arriba, construida en conjunto y en defensa de aquellos que usan $\operatorname{drogas}^{(9,10)}$.

En ese sentido, este artículo tiene como objetivo discutir el consumo del virado como estrategia de reducción de daños entre personas que usan crack, práctica identificada en estudios anteriores realizados en Pernambuco. El virado consiste en transformar la piedra del crack en un polvo, al que se le adiciona ácido bórico para ser aspirado. Al calentar el polvo de la piedra del crack junto al ácido bórico se obtiene el borato de cocaína, un polvo blanco que se aspira y se absorbe por la mucosa nasal ${ }^{(11)}$.

Los datos provienen de una investigación exploratoria mayor, con abordaje cualitativo, que aborda los aspectos relacionados con la cultura del uso del crack en el estado de Pernambuco, y se titula "Vulnerabilidade de usuários de crack ao HIV e outras doenças transmissíveis: estudo sociocomportamental e de prevalência no estado de Pernambuco", realizado en el marco del Instituto Aggeu Magalhães, Fundação Oswaldo Cruz, Pernambuco.

En un artículo anterior derivado de esa investigación, se analizaron aspectos que los usuarios señalaron como puntos importantes para el tratamiento: la voluntad, la inclusión de la espiritualidad, la diversidad en la oferta de actividades, el espacio protegido de la droga y la formación profesional con inclusión socioproductiva ${ }^{(12)}$.

Otro estudio publicado sobre la misma investigación, señalaba que el uso problemático de crack, que se relaciona fuertemente con la exclusión social, incurre en situaciones de marginalidad asociadas a la cultura del uso y promueve ambientes conflictivos, violentos e insalubres. El sentimiento de pérdida de identidad, derivado del estigma de la droga, también estuvo presente en el relato de los entrevistados ${ }^{(13)}$. Además, se publicó otro estudio sobre la historia de violencia entre mujeres que usan crack $^{(14)}$.

\section{METODOLOGÍA}

Con el propósito de comprender las dinámicas sociales relacionadas con la cultura de uso del crack $^{(15)}$, se optó por un abordaje cualitativo, que permite describir y analizar culturas y comportamientos desde el punto de vista de las personas estudiadas ${ }^{(16)}$, identificando valores, creencias, opiniones y comportamientos $^{(17,18)}$.

Entre marzo y agosto de 2016, se realizaron entrevistas semiestructuradas a 39 personas (hombres, mujeres y travestis), mayores de 18 años, que usaban crack regularmente (al menos 25 días en los últimos seis meses) y que eran atendidas en los cuatro núcleos regionales (Recife, Jaboatão dos Guararapes, Cabo de Santo Agostinho y Caruaru) del Programa Actitud, Programa de Atención Integral a los Usuarios de Drogas y sus Familiares, de la Secretaría Ejecutiva de Políticas sobre Drogas de Pernambuco.

El Programa Actitud integra la Política Estadual de Asistencia Social y tiene como objetivo responder a la situación de vulnerabilidad social de usuarios de drogas y sus familiares. Actualmente, es referencia en el cuidado de los usuarios de crack y otras drogas en Pernambuco ${ }^{(19)}$, y atiende prioritariamente a aquellas personas en situación de exposición a la violencia, que necesitan alejarse de sus comunidades, cuyos vínculos familiares son frágiles o se han roto.

Como los autores de este estudio no tenían vínculos con el Programa Actitud, para convocar a las personas se contó con el apoyo de un equipo de profesionales, que desempeñó la función de gatekeepers, es decir, conscientes de los criterios de inclusión, identificaron a personas que potencialmente podrían hablar sobre el tema y que, el día de la entrevista no estuvieran bajo los efectos del crack o de otras drogas. Luego de convocar a los posibles participantes, se los invitaba a participar del estudio, se les explicaban los objetivos y se solicitaba la firma del consentimiento libre e informado. Se sustituyeron los nombres de los entrevistados por nombres de piedras preciosas a fin de preservar su 
identidad, y garantizar también la confidencialidad de los datos.

Se realizaron entrevistas semiestructuradas con una guía elaborada sobre la base de la bibliografía de la cultura de uso de crack, que abordó diferentes temáticas. En este artículo, se presentan los resultados referentes a la discusión del uso del virado como estrategia de reducción de daños entre los usuarios de crack.

La autora principal realizó todas las entrevistas y, con el propósito de reducir las variaciones en la forma de transcribir el relato y disminuir los posibles sesgos en el análisis, una sola profesional capacitada realizó las transcripciones. Además, como procedimiento de validación de las entrevistas, las transcripciones se compararon, en su totalidad, con el audio original. En la preparación del material para su posterior análisis, se utilizó el software NVivo 10.0, actualmente la herramienta más utilizada para el análisis de datos cualitativos de los artículos indizados en la base de datos PubMed.

Una vez transcriptas las entrevistas se aplicó la técnica de análisis de contenido, basado en las definiciones de Bardin ${ }^{(20)}$. Para la autora, el procedimiento es un conjunto de técnicas, que permean el análisis cualitativo y que consisten en la explicitación y sistematización del contenido de los mensajes para generar deducciones lógicas y justificadas, para lo cual se realizaron las tres etapas recomendadas por el método: preanálisis, exploración del material y tratamiento de los resultados.

Inicialmente se realizaron lecturas exhaustivas de las entrevistas para comprender en profundidad la experiencia vivida por cada uno de los participantes, e identificar los ejes temáticos. Luego, se atribuyeron los primeros significados a los resultados brutos analizados. Este proceso se realizó a través de la categorización, que permitió la clasificación de los elementos de significación, cuya técnica consiste en calificar los diferentes elementos según núcleos de sentido ${ }^{(20)}$. Las categorías emergen de los patrones y temas identificados en los datos recolectados, del significado principal observado en el material explorado por el análisis de contenido ${ }^{(18)}$.
De esta forma, en los resultados se presentan los ejes temáticos y sus respectivas categorías de análisis. En el presente artículo, el eje temático analizado fue el uso del virado y la reducción de daños, y se identificaron las siguientes categorías: escapar al estigma social; uso controlado del virado para una mayor sociabilidad; aumento de la libido; y riesgo de transmisión de enfermedades infecciosas.

El proyecto fue aprobado por el comité de ética en investigación del Instituto Aggeu Magalhães, Fundação Oswaldo Cruz Pernambuco (CAE 25250413.6.0000.5190) y de la Universidade Federal de São Paulo (CAAE 33243514.3.0000.5505).

\section{RESULTADOS Y DISCUSIÓN}

Entre las formas identificadas de consumo del crack, el virado fue uno de los hallazgos importantes, tanto en el presente estudio, como en otras investigaciones llevadas a cabo en Pernambuco, que configuraron un gran diferencial en la cultura de uso del estado ${ }^{(11,21,22)}$

El uso del crack bajo la forma de virado ha sido mencionado con frecuencia entre usuarios de crack de Pernambuco. En el informe del estudio epidemiológico desarrollado con 1.062 usuarios del estado, el $54,3 \%$ relató que ya había consumido crack de esa forma ${ }^{(22)}$. Esos datos corroboran los hallazgos de Nappo et al. ${ }^{(11)}$, que señalan un aumento significativo de esta forma de uso en Pernambuco desde su identificación. Es importante destacar que estos estudios también señalan que el consumo del virado es característico de este estado, y este uso no ha sido registrado, aún, en ningún otro lugar de Brasil o del exterior.

\section{Escapar al estigma social}

En el transcurso de las entrevistas, tuvimos la posibilidad de comprender mejor las formas de uso y, sobre todo, identificar posibles diferencias entre el crack y el virado. ¿Cuándo 
optaban por el crack y cuándo por el virado?, ¿el virado sería una estrategia de protección frente a los riesgos observados por un uso compulsivo, característico del consumo del crack? En este sentido, en el siguiente relato es posible diferenciar algunos aspectos del crack y del virado para comprender mejor no solo el contexto de uso, sino también las especificidades de cada forma de usar esta sustancia.

No tiene el sabor que sentís cuando fumás la piedra, cuando aspirás la piedra no vas a sentir el sabor, vas a sentir un ardor en la nariz. No te va a saciar, esa sensación de saciedad de cuando fumás una piedra. Te va a mantener muy despierto, demasiado enérgico, pero no "noiado" [paranoide] como quedamos nosotros... La sensación cuando aspirás el virado es sentirte más enérgico, más potente, más valiente... Con el crack, sentís miedo, te sentís arrinconado. No salís de tu lugar. No querés que nadie te mire. Sentís vergüenza porque sos "noiada". Cuando pasan las personas, te miran, y te ven realmente como un "noiado". Quedás descontrolado. Esas son las diferencias. (Lodolita, travesti, 20 años)

En este relato, Lodolita diferencia el crack y el virado a partir del estigma establecido para las personas que usan crack. El estigma puede ser considerado una marca física o social de connotación negativa, que lleva al portador de esa marca a ser marginalizado o excluido de algunas situaciones sociales. Así, el estigma puede ser considerado como una construcción social de una marca que le confiere a la persona un estatus desvalorizado cuando se lo compara con otros miembros de la sociedad. Esto se produce cuando a los individuos se los identifica con alguna característica negativa que poseen $y$, a partir de ese momento, pasan a ser discriminados y desvalorizados por la sociedad. A ese proceso se lo denomina estigma social o público ${ }^{(23)}$.

Según Ronzani, Noto y Silveira ${ }^{(23)}$, las personas que usan drogas, especialmente crack, sufren diariamente las consecuencias de ese procesos de estigmatización, que se evidencian socialmente tanto en la pérdida de la autoestima, la restricción de las interacciones sociales, una menor percepción de los problemas que necesitan atención, como en las limitadas perspectivas ante un proceso de recuperación, que influyen negativamente en el tratamiento de esas personas.

Almeida ${ }^{(24)}$ reafirma que las posturas cargadas de preconceptos y moralistas crean una representación de las personas que usan drogas como criminales, peligrosas y sin carácter, estigmatizándolas y apartándolas de los servicios o de cualquier persona que pueda ofrecer algún tipo de cuidado. Ese estigma también aumenta la dificultad de abordar el tema y que las personas que tienen una relación perjudicial con las drogas puedan ayudarse entre sí, construyéndose un círculo vicioso.

Además, la información engañosa publicada por los medios, más allá de la falta de conocimientos sobre el trastorno, genera temor acerca de las personas que usan drogas y se las considera incapaces de recuperarse. Así, viven un proceso de sufrimiento frente a la desconfianza basada en estereotipos negativos, en preconceptos y en procesos de discriminación. Según Petuco ${ }^{(25)}$, en las páginas de los grandes periódicos, o incluso en la televisión y en las campañas de prevención, se identifica al "usuario de crack" con la figura de un monstruo deshumano e irracional.

El "noia" [forma reducida de paranoia] es aquella persona que no respeta las normas sociales y los parámetros éticos en busca de una piedra de crack, se la ve desconfiada y no consigue establecer otro tipo de relaciones con las personas ${ }^{(26)}$. En ese sentido, Merhy ${ }^{(27)}$ afirma que

Hoy, hay sectores conservadores que están haciendo un enorme esfuerzo para construir un imaginario social que visibilice a los usuarios de drogas como zombis, no humanos. Como victimizados por la captura-dependencia que las substancias químicas ilícitas les provocarían, de tal manera que dejaron de ser sujetos deseantes para ser meros objetos 
inertes e irresponsables, respecto de sus propios actos.

Ese lugar estigmatizante, de ser visto como un zombi o incluso como un no humano, no es aceptado por muchas de las personas que usan crack. Estar en esa posición significa haber fracasado, haber negado su condición humana, y la estrategia de protección de usar crack en forma de virado surge como una posibilidad de cuidado, y como una estrategia de reducción de daños, construida a partir de la experiencia de atreverse a otras formas de consumo de una misma sustancia.

\section{El uso controlado del virado para una mayor sociabilidad}

En esta categoría, el uso del virado surge como una estrategia de reducción de daños para evitar el uso compulsivo de crack. Ciertos estudios muestran que, frente a las especificidades psicofarmacológicas del crack, el patrón predominante de uso de esta droga es del tipo abusivo, o sea, el usuario compulsivo utiliza grandes cantidades de la sustancia en un corto lapso de tiempo. Los usuarios de crack tienen la tendencia de usar el crack por horas o incluso días de forma intermitente, y si bien pueden alternar días sin uso que pueden durar hasta incluso meses enteros, siempre tienen episodios de uso intensivo ${ }^{(28,29)}$. Entre los patrones de uso identificados, el consumo compulsivo fue el más relatado, que se caracteriza por un uso diario de crack que puede extenderse a lo largo del día, y que se detiene solo cuando el usuario alcanza el agotamiento físico, psíquico o financiero(30).

El virado te deja más ligado. No conseguís dormir. Aspirás, tomás cerveza, y amanece. Ya pasé tres días y tres noches aspirando virado. El virado, creo, hace menos daño, creo que es menos. La paranoia es menor que cuando fumás. Con el virado te vinculás, conversás. Con el crack no. Fumás y no querés hablar con nadie, te quedás mudo, amedrentado. Con el virado, no, estás vinculado, aspirás, con- versás, jugás, pero el crack no te hace ese efecto, no. (Granada, hombre, 27 años)

No usás el virado todo el día, no salís a robar, lo usás más los fines de semana. Si salimos y vamos a bailar, llevo polvo para aspirar y ahí lo aspirás y al otro día estás normal. No querés seguir usándolo. Lo usás hoy, y mañana ya no lo querés usar más. El crack no, cuanto más dinero tenés para gastar, pasás dos, cuatro días sin dormir. (Esmeralda, hombre, 21 años)

Los relatos anteriores muestran que el uso del virado se da en ambientes de fiestas y diversión. Al compararlo con el crack, los entrevistados mencionan que el virado te deja "despierto", "enérgico", sin sentir la paranoia del crack. El uso del virado es más sociable, los usuarios no buscan el aislamiento como sucede con el crack. El uso compulsivo del crack no se identifica con el uso del virado.

Esta relación compulsiva de las personas que usan crack fue identificada por $\mathrm{Nappo}^{(1)}$ y Oliveira ${ }^{(31)}$ y afirman que este patrón de consumo se instala luego de la experimentación. En la investigación de Oliveira ${ }^{(31)}$, varios entrevistados ignoraban las necesidades básicas como alimentación, sueño e higiene en función del uso de la droga. También relataron el empeoramiento de la calidad de vida al priorizar el consumo de crack por sobre todas las otras actividades.

Leite, Oliveira y $\mathrm{Cruz}^{(30)}$ también identificaron el patrón de uso compulsivo entre sus entrevistados, quienes describieron el uso del crack de manera frecuente e intensa, limitando sus rutinas diarias hasta dedicarse exclusivamente al uso de la droga. Para estos investigadores, describir esa experiencia es adentrarse en la compulsión y la falta de control frente al crack, es ver cómo desaparece la libertad de elección que da lugar al consumo cada vez mayor de la droga, por más que el cuerpo no lo tolere.

En los relatos se percibe que las personas entrevistadas conocen esa posibilidad de uso compulsivo del crack y terminan optando por el uso del virado para poder mantener una vida más sociable y placentera, lo que 
refuerza la posibilidad del uso del virado como una estrategia de reducción de daños.

La diferencia es que, al inhalar el humo, te pega en el momento. El impacto es que quedás paralizado en el momento, te paralizás, te pegás un viaje de cinco segundos, después de esos cinco segundos, quedás un poco asustado y un poco nervioso. En cambio, el virado tarda un poco para que te pegue, hasta que se diluya en el cuerpo, Ilegue a la mente, Ilegue a aquella celulita que pega más, tarda un poco más... (Ágata, Hombre, 34 años)

En el relato, el entrevistado distingue bien la sensación del crack y del virado. Esa diferenciación se basa en la forma de ingerir el mismo principio activo de la cocaína. Las drogas fumadas tienen un efecto inicial más rápido y con duración más efímera. Las drogas aspiradas demoran más, tanto el inicio del efecto como su permanencia en el organismo. Según Nappo(1), en las drogas fumadas, la absorción del gas se da a través del pulmón, órgano con una gran área de absorción, por lo que rápidamente llega al sistema nervioso central, por el camino más corto. Cuando la droga es aspirada, como el virado, la absorción se da por la mucosa nasal, que es más lenta y demora más tiempo para llegar al sistema nervioso central y en ese trayecto sufre la acción de enzimas que la destruyen, por lo que solo una pequeña parte de lo aspirado llega al cerebro.

El crack es una droga que, cuando se fuma, produce una gran euforia; sin embargo, esa sensación tiene corta duración, seguida de una voluntad casi incontrolable de usar la sustancia una vez más, lo que caracteriza la fisura. Entre las personas que tienen usos problemáticos de crack, es común la dificultad de controlar el uso, por lo que pasan días y noches consumiendo la droga ${ }^{(28)}$.

Sin embargo, también es común observar a personas que consumen drogas como el crack y que utilizan estrategias para mermar los efectos y disminuir los eventuales daños ocasionados por el uso, como ingerir líquidos, alimentarse antes del uso, dormir, intercalar o asociar el crack con drogas consideradas más leves, como la marihuana, el cigarrillo y el alcohol ${ }^{(29)}$.

En el contexto de este estudio, probablemente el uso del virado tenga la misma connotación de creer que se trata de una droga más leve, con efecto más duradero y sociable, sin contar con el estigma social en el cotidiano del consumo de la droga.

\section{El aumento de la libido}

En esta categoría se pudieron analizar las diferencias de comportamiento sexual de una de las entrevistadas bajo los efectos del crack y del virado. El comportamiento sexual puede ser entendido como un conjunto de características relacionadas con el tipo de compañía, el número de parejas sexuales, la relación establecida con la pareja, entre otras $^{(32)}$. En el discurso de la usuaria se percibe que, bajo el efecto del crack, el tipo de vínculo era exclusivamente comercial y las relaciones establecidas con sus parejas tenían un interés específico: obtener droga, sin necesariamente sentir placer sexual. La falta de libido puede influir en el descuido del propio cuerpo $^{(33)}$ y, en consecuencia, en la realización de prácticas sexuales menos seguras.
La diferencia del polvo con el crack, para mí, es que con el polvo conseguís tomar, conseguís hacer el amor, conseguís tener relaciones, estás más eléctrica. Con el crack no. El crack te quita el deseo, te causa impotencia sexual. No tenés sexo con placer. Si lo hacés, lo hacés por dinero, hasta para consumir droga, como ya lo hice muchas veces, pero placer no dá. (Ouro, mujer, 32 años)

El relato de Ouro advierte sobre el uso del crack bajo la forma de virado, como estrategia de aumento de la libido y mayor interacción con sus compañeros en las relaciones sexuales, considerando el hecho de que el virado provoca un efecto de euforia, opuesto a la paranoia provocada por el crack. Esta forma de uso podría llevar a comportamientos 
sexuales con menores riesgos de transmisión del VIH y de otras infecciones sexualmente transmisibles, que de acuerdo con la bibliografía, las prácticas sexuales desprotegidas están directamente relacionadas con el tipo de relación establecida con el compañero, el tipo de compañía y el patrón de uso compulsivo de la droga ${ }^{(34,35)}$.

\section{Riesgo de transmisión de enfermedades infecciosas}

Otro aspecto importante de este estudio es la cuestión del sangrado nasal, muy observada en el uso de virado, que señala que, cuando se comparten objetos para el consumo de la droga, existe un riesgo mayor de transmisión de enfermedades infecciosas, como el VIH y la hepatitis C. El canuto que se utiliza para inhalar el virado, normalmente es de uso compartido. Esa práctica expone a las personas a un mayor riesgo.

Esta categoría dialoga con las estrategias de reducción de daños establecidas por las personas que usan crack, dado que el consumo de virado expone a esas personas a un riesgo mayor, y necesitan orientación específica para esta forma de consumo del crack. Esta acción de prevención se torna imprescindible dado que, en los relatos de los entrevistados, no se identificó mención alguna al riesgo de infección por VIH u otra enfermedad, o incluso alguna referencia a formas de evitar compartir canutos.

La diferencia es que a algunas personas puede causarle sangrado en la nariz, al no estar acostumbrado. En mi caso, la primera vez que aspiré virado, lo di vuelta y otras personas también, entonces cuando aspiré por primera vez, ahí mi nariz sangró. (Ágata, hombre, 34 años)

Compartir los canutos u otros utensilios siempre fue una práctica muy utilizada entre las personas que consumen cocaína. Según Niel y Silveira ${ }^{(36)}$, al utilizar la cocaína en polvo, esparcen la droga en una superficie plana, la dividen en pequeñas hileras y la aspiran a través de un objeto similar a un canuto, hecho de billetes, hojas de papel o canutos de plástico. A pesar de que es menos común, existe también la posibilidad de que depositen el polvo directamente en la región de la mano ubicada entre los dedos índice y pulgar, y lo acercan directamente a la nariz para aspirar. Según los autores, la práctica de compartir los utensilios para el uso de drogas aumenta el riesgo de infección, incluso del VIH. De esta forma, en lugar de billetes, o cualquier otro tipo de papel, se orientaba a las personas a dar preferencia a los canutos de plástico que se distribuían en los kits de reducción de daños y a no compartir los canutos, dado que esta sustancia, al ser un potente vasoconstrictor, podría provocar el sangrado nasal, facilitando así la transmisión de enfermedades.

Estas orientaciones no están dirigidas a las personas que usan virado, porque la gran preocupación es el uso del crack fumado. La gran mayoría de las estrategias de reducción de daños desarrolladas para las personas que usan crack están relacionadas al "tiro en la lata", o sea, al consumo en latas de gaseosa, $y$ al uso de preservativo.

Las personas que usan drogas construyen estrategias a partir de los intercambios sociales que ocurren en el momento del uso. Esas posibilidades de consumo no surgen de manera aislada, ni independiente de los efectos de la sustancia utilizada, ni tampoco de las construcciones colectivas, fruto de la dinámica social establecida entre el grupo, lo que puede viabilizar formas de uso menos dañinas ${ }^{(37)}$.

\section{CONSIDERACIONES FINALES}

La realización de esta investigación tuvo como objetivo analizar el uso del virado como estrategia de reducción de daños entre las personas que usan crack en el Programa Actitud, estado de Pernambuco, para que nuevas intervenciones puedan ser pensadas a partir de las experiencias de los entrevistados en nuestra región. Si bien no hubo ninguna 
intención de generalizar esas experiencias, creemos que la singularidad pueda relacionarse con otras personas involucradas en la misma problemática. Así, comprendemos que las experiencias de estos usuarios, aunque singulares, puedan propagarse entre los profesionales que actúan en esas áreas. Ese es un desafío a ser enfrentado por todos los que trabajan con usuarios de drogas, principalmente, de crack, en el sentido de garantizarles un cuidado adecuado a sus demandas.

Ante las cuestiones planteados en este estudio, resulta imprescindible el conocimiento de la cultura del uso de drogas en diferentes contextos para planear y desarrollar acciones de atención a la salud, que incluyan acciones de prevención. El estudio señaló la necesidad de comprender no solo las formas de consumo del crack y sus estrategias de reducción de daños, sino también los significados que esas estrategias tienen para cada persona que usa crack.

Se identificó que el uso del crack en forma de virado fue señalado por algunos participantes del estudio como una estrategia de protección contra el estigma social, comúnmente dirigido a personas que usan crack de forma compulsiva, así como para controlar el uso compulsivo, mantener la libido y la convivencia social.

Por otro lado, si se comparten los utensilios, usar crack en forma de virado representa un mayor riesgo de transmisión de enfermedades infecciosas como el VIH y hepatitis C. Sin embargo, los entrevistados no mostraron conocer esta forma de infección lo que reafirma la necesidad de que los profesionales se sumerjan en la cultura de uso del crack en sus diversas formas de consumo y en diferentes contextos.

\section{AGRADECIMIENTO}

La investigación titulada "Vulnerabilidade de usuários de crack ao HIV e outras doenças transmissíveis: estudo sociocomportamental e de prevalência no estado de Pernambuco" fue financiada por la Secretaria de Vigilância em Saúde, Ministério da Saúde a través de la convocatoria $20 / 2013$

A todas las colaboraciones institucionales: Programa Atitude dependiente de la Secretaria Executiva de Políticas Sobre Drogas, Secretaria de Desenvolvimento Social, Criança e Juventude del Gobierno de Pernambuco; a los programas estaduales de salud de Pernambuco: Programa Estadual de DST/Aids y Programa de Controle da Tuberculose; Laboratório Central de Saúde Pública, Secretaria Estadual de Saúde de Pernambuco; Secretaria Municipal de Saúde, Cabo de Santo Agostinho; Secretaria de Saúde de Caruaru; Secretaria Executiva de Promoção da Saúde, Secretaria Municipal de Desenvolvimento e Mobilização Social de Jaboatão dos Guararapes; Secretaria de Saúde do Recife; Centro Brasileiro de Informações sobre Drogas Psicotrópicas- Universidade Federal de São Paulo (CEBRID-UNIFESP).

\section{REFERENCIAS BIBLIOGRÁFICAS}

1. Nappo AS. "Baquêros" e "Craquêros: um estudo sobre o consumo de cocaína na cidade de São Paulo. [Tese doutorado]. São Paulo: Universidade Federal de São Paulo, Escola Paulista de Medicina; 1996.

2. Bastos Fl, Bertoni N, (orgs.). Pesquisa nacional sobre o uso de crack: quem são os usuários de crack e/ou similares do Brasil? quantos são nas capitais brasileiras? Rio de Janeiro: ICICT, Fiocruz; 2014

3. Bastos Fl, Cotrim BC. O consumo de substâncias psicoativas entre os jovens brasileiros: dados, danos e algumas propostas. En: Jovens acontecendo na trilha das políticas públicas. Tomo 2. Brasília: CNPD; 1998. p. 645-670.

4. Zaluar A. Integração perversa: pobreza e tráfico de drogas. Rio de Janeiro: FGV; 2004.

5. Dias AC, Araújo MR, Dunn J, Sesso RC, Castro V, Laranjeira R. Mortality rate among crack/cocaine-dependent patients: A 12-year prospective cohort study conducted in Brazil. Journal of Substance Abuse Treatment. 2011;41(3):273-278. doi:10.1016/j.jsat.2011.03.008

6. Petuco DRS. O pomo da discórdia?: Drogas, saúde, poder. Curitiba: CRV; 2019.

7. Machado LV, Boarini ML. Políticas sobre drogas no Brasil: a estratégia de redução de danos. Psicologia: Ciência e Profissão. 2013:33(3):580-595. doi: 10.1590/ S1414-98932013000300006. 
8. Ayres JRCM, Calazans GJ, Saletti Filho HG, Franca Junior I. Risco, vulnerabilidade e práticas de prevenção e promoção da saúde. En: Campos GWS, Minayo MCS, Akerman M, Drumond Júnior M, Carvalho YM, (orgs.). Tratado de saúde coletiva. São Paulo, Hucitec; 2006. p. 375-417.

9. Dias JC, Scivoletto S, Silva CJ, Laranjeira RR, Zalesk M, Gigliotti A. et al. Redução de danos: posições da Associação Brasileira de Psiquiatria e da Associação Brasileira para Estudos do Álcool e Outras Drogas. Jornal Brasileiro de Psiquiatria. 2003;52(5):341-348.

10. Cruz MS, Sáad AC, Ferreira SMB. Posicionamento do Instituto de Psiquiatria da UFRJ sobre as estratégias de redução de danos na abordagem dos problemas relacionados ao uso indevido de álcool e outras drogas. Jornal Brasileiro de Psiquiatria. 2003;52(5):355-362.

11. Nappo SA, Sanchez ZM, Rameh R, Almeida R, Uchôa R. Virado: a new method of crack consumption in Brazil. American Journal on Addictions. 2012;21(6):574575. doi: 10.1111/j.1521-0391.2012.00272.x.

12. Almeida RBF, et al. O tratamento da dependência na perspectiva das pessoas que fazem uso de crack. Interface - Comunicação, Saúde, Educação. 2018;22(66):745756. doi: 10.1590/1807-57622016.0940.

13. Almeida RBF, Santos NTV, Brito-Silva KS, Brito AM, Nappo SA. A cultura de uso de crack em Pernambuco: um estudo sobre lugares e sentimentos dos invisíveis. En: Figueiredo GLA; Martins CHG, Akerman M, (orgs.). Vulnerabilidade e saúde: grupos em cena por visibilidade no espaço urbano. São Paulo: Hucitec; 2018. p. 188-210.

14. Santos DP, Jacques IJAA, Diniz GTN, Brito AM, Santos NTV. Histórico de violência entre mulheres que fazem uso de crack no estado de Pernambuco, Brasil. Saúde em Debate. 2018;42(119):862-875. doi: 10.1590/0103-1104201811906.

15. Malchy L, Bungay V, Johnson J. Documenting practices and perceptions of "safer" crack use: A Canadian pilot study. International Journal of Drug Policy. 2008;19:339-341. doi: 10.1016/j.drugpo.2007.06.005.

16. World Health Organization. WHO initiative on cocaine: meeting of project advisers, Geneva, 24-28 August 1992 [Internet]. 1992 [citado 10 may 2019]. Disponible en: https://tinyurl.com/ydbz2ax7.

17. Taylor SJ, Bodgan R. Introduction to qualitative research methods. New York: John Wiley \& Sons; 1998.

18. Patton MQ. Qualitative research and evaluation methods. 3rd ed. Saint Paul: Sage Publications; 2002.

19. Ratton JL, (coord.). Políticas de drogas e redução de danos no Brasil: o Programa Atitude em Pernambuco. Recife: NEPS, UFPE; 2016.

20. Bardin L. Análise de conteúdo. São Paulo: Edições 70; 2011.

21. Santos NTV. Vulnerabilidade e prevalência de HIV e sífilis em usuários de drogas, Recife, 2009: resultados de um estudo respondente-drivensampling. [Tese doutorado]. Recife: Cento de Pesquisa Aggeu Magalhães, Fundação Osvaldo Cruz; 2013.

22. Santos NTV, Almeida RBF, Brito AM. Vulnerabilidade de usuários de crack a HIV e outras doenças transmissíveis: estudo sóciocomportamental e de prevalência no estado de Pernambuco. Recife: Centro de Pesquisa Aggeu Magalhães, Fundação Oswaldo Cruz; 2016.

23. Ronzani TM, Noto AR, Silveira PS. Reduzindo o estigma entre usuários de drogas. Guia para profissionais e gestores. Juiz de Fora: Editora UFJF; 2014.

24. Almeida RBF. Drogas, estigma e vulnerabilidade: conhecer para prevenir. En: Valois-Santos NT, Almeida RBF, Oliveira EM, (org.). Apostila do Curso de Atualização para Qualificação da Rede de Atenção Integral a Pessoas que Fazem Uso de Drogas. Recife: Departamento de Saúde Coletiva, Instituto Aggeu Magalhães, Fiocruz; 2017. p. 80-101.

25. Petuco DRS. Entre imagens e palavras: o discurso de uma campanha de prevenção ao crack. [Dissertação mestrado]. Paraíba: Universidade Federal da Paraíba; 2011.

26. Gomes BR, Adorno RCF. Tornar-se "noia": trajetória e sofrimento social nos "usos de crack" no centro de São Paulo. Etnográfica. 2011;15(3):569-586. doi: 10.4000/ etnografica.1076.

27. Merhy E. Anormais do desejo: os novos não humanos?: Os sinais que vêm da vida cotidiana e da rua. En: Conselho Federal de Psicologia, Grupo de Trabalho de Álcool e outras Drogas. Drogas e cidadania: em debate. Brasília: CFP; 2012. p. 9-18.

28. Oliveira LG, Nappo SA. Caracterização da cultura do crack na cidade de São Paulo: Padrão de uso controlado. Revista de Saúde Pública. 2008;42(4):664-667. doi: 10.1590/S0034-89102008005000039.

29. Ribeiro LB, Sanchez ZM, Nappo SA. Estratégias desenvolvidas por usuários de crack para lidar com os riscos decorrentes do consumo da droga. Jornal Brasileiro de Psiquiatria. 2010;59(3):210-218. doi: 10.1590/S004720852010000300007.

30. Leite SC, Oliveira MM, Cruz VC. O encontro com o crack: inicio, tempo, quantidade diária e formas de uso. SMAD: Revista Eletrônica Saúde Mental Álcool e Drogas. 2015;11(2):97-104.

31. Oliveira LG. Avaliação da cultura do uso de crack após uma década de introdução da droga na cidade de São Paulo. [Tese doutorado]. São Paulo: Universidade Federal de São Paulo, Escola Paulista de Medicina, Programa de Pós-Graduação em Psicobiologia; 2007.

32. Berquó E, (coord.). Comportamento sexual da população brasileira e percepções do HIV/Aids. Brasília: Ministério da Saúde, CEBRAP; 2000.

33. Paranhos RFB, Paiva MSC, Evanilda SS. Vivência sexual e afetiva de mulheres com incontinência urinária secundária ao HTLV. Acta Paulista de Enfermagem. 2016;29(1):47-52. doi: 10.1590/1982-0194201600007. 
34. Malta M, Monteiro S, Lima RMJ, Bauken S, Marco A, Zuim GC, Bastos Fl, Singer M, Strathdee SA. HIV/Aids risk among female sex workers who use crack in Southern Brazil. Revista de Saúde Pública. 2008;42(5):830-837. doi: 10.1590/S0034-89102008000500007.

35. Nappo SA, Sanchez Z, Oliveira LG. Crack, AIDS and women in São Paulo, Brazil. Substance Use \& Misuse. 2011;46(4):476-485. doi: 10.3109/10826084. 2010.503480
36. Niel M, Silveira DX. Drogas e redução de danos: uma cartilha para profissionais de saúde. São Paulo: PROAD, UNIFESP, Ministério da Saúde; 2008.

37. Jorge MSB, Quinderé PHD, Yasui S, Albuquerque RA. The ritual of crack consumption: socio-anthropological aspects and impacts on the health of users. Ciência \& Saúde Coletiva. 2013;18(10):2909-2918. doi: 10.1590/s1413-81232013001000015.

\section{FORMA DE CITAR}

Almeida RBF, Santos NTV, Brito AM, Silva KSB, Jacques IJAA, Nappo SA. EI uso de "virado" como estrategia de reducción de daños entre los usuarios de crack del estado de Pernambuco, Brasil. Salud Colectiva. 2020;16:e2528. doi: 10.18294/sc.2020.2528.

Recibido: 01 sep 2019 | Versión final: 3 abr 2020 | Aprobado: 14 abr 2020 | Publicado en línea: 16 may 2020

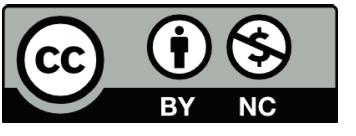

Esta obra está bajo una licencia de Creative Commons Reconocimiento-NoComercial 4.0 Internacional. Reconocimiento - Permite copiar, distribuir y comunicar públicamente la obra. A cambio, se debe reconocer y citar al autor original. No Comercial - Esta obra no puede ser utilizada con finalidades comerciales, a menos que se obtenga el permiso. 